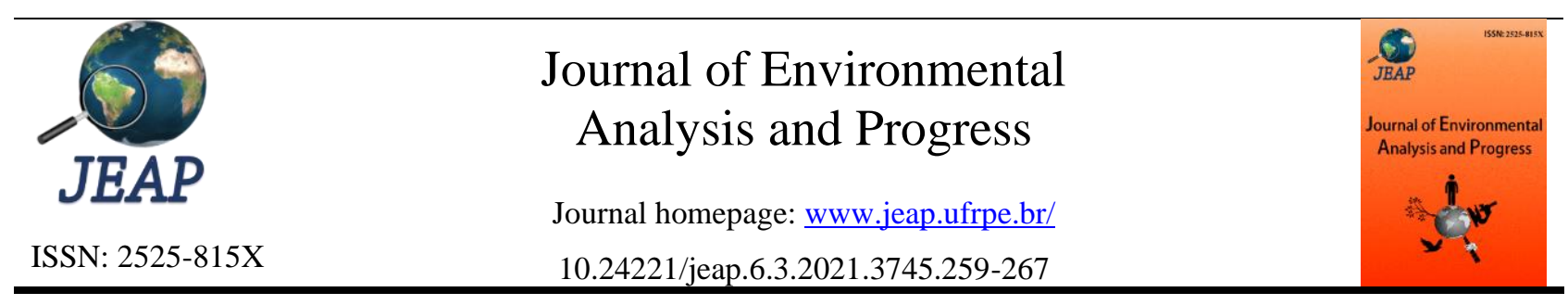

\title{
Avaliação de rúmen bovino como inóculo de reatores anaeróbios
}

\section{Evaluation of bovine rumen as inoculum of anaerobic reactors}

\author{
Rachel Barros Pires ${ }^{\mathrm{a}}$, Emanuele Diógenes Guerra ${ }^{\mathrm{a}}$, Luiza Feitosa Cordeiro de Souza ${ }^{\mathrm{b}^{*}}$, Simone \\ Machado Santos ${ }^{\mathrm{a}}$ \\ ${ }^{a}$ Universidade Federal de Pernambuco-UFPE, Campus Agreste-CAA. Av. Campina Grande, s/n, Km 59, Nova Caruaru, \\ Caruaru-PE, Brasil. CEP: 55014-900. E-mail: rachelb.pires@ outlook.com, manu diogenes@hotmail.com, \\ smachados@hotmail.com. \\ b Centro Universitário Tabosa de Almeida-ASCES-UNITA. Av. Portugal, n. 584, Bairro Universitário, Caruaru-PE, \\ Brasil. CEP: 55016-901. E-mail: luizas@ gmail.com*.
}

\begin{tabular}{l}
\hline A R T I C L E I N F O \\
\hline Recebido 29 Jul 2020 \\
Aceito 18 Ago 2021 \\
Publicado 14 Set 2021
\end{tabular}

\begin{abstract}
A B S T R A C T
Bovine rumen is a source of microorganisms that perform the anaerobic digestion of carbon compounds. Its high cellulolytic activity accelerates the start-up in anaerobic reactors, which is usually slow and unstable. This study aimed to evaluate the potential of using bovine rumen as an inoculum in anaerobic reactors. The test was performed in sequential batches, bench scale, static regime, and temperature control of $30^{\circ} \mathrm{C}$. The reactors were fed with a solution of volatile fatty acids of $6000 \mathrm{mg} \mathrm{O} 2$ $\mathrm{L}^{-1}$ (COD) and 3 g SSV.L $\mathrm{L}^{-1}$ of the respective biomasses: the raw rumen, washed rumen, and brewery sludge. The COD degradation, methane produced, and each biomass's production speed was measured and analyzed. After the experimental period, it was observed that despite the bovine rumen having a higher concentration of volatile solids, its methane production was lower when compared to the brewery sludge. However, the rumen showed better COD degradation. The Specific Methanogenic Activity (SMA) for the washed and raw rumens was 9 and 4 times lower than the brewery sludge in the first stage. In the second stage, there was an increase of 2.4 and 2.7 times in SMA for the washed and crude rumen, respectively, compared with the previous stage. It is concluded that bovine rumen can be used to inoculate an anaerobic biological reactor to degrade organic matter and methane production as biogas.
\end{abstract}

Keywords: SMA, anaerobic digestion, inoculum, rumen.

\section{R E S U M O}

O rúmen bovino é uma fonte de microrganismos que realizam a digestão anaeróbia de compostos de carbono. A sua alta atividade celulolítica acelera o processo de partida em reatores anaeróbios, que tende a ser lento e instável, quando realizado sem inóculo. O estudo objetivou avaliar o potencial do uso de rúmen bovino como inóculo de reatores anaeróbios. O teste foi realizado em bateladas sequenciais, escala de bancada, regime estático e com controle de temperatura de $30^{\circ} \mathrm{C}$. Os reatores foram alimentados com uma solução ácidos graxos voláteis de $6000 \mathrm{mg} \mathrm{O}_{2} \mathrm{~L}^{-1}$, de DQO e $3 \mathrm{~g} \mathrm{SSV} . \mathrm{L}^{-1}$ das respectivas biomassas, rúmen bruto, rúmen lavado e lodo de cervejaria. O desempenho da degradação da DQO, a quantidade de metano produzida e a velocidade de produção de cada biomassa foram medidos e analisados. Após o período experimental, observou-se que apesar do rúmen bovino ter maior concentração de sólidos voláteis, a sua produção de metano foi menor, quando comparado ao lodo de cervejaria. Entretanto, o rúmen apresentou melhor desempenho na degradação da DQO. A Atividade Metanogênica Específica (AME) para os rúmenes lavado e bruto foram nove e quatro vezes inferior ao do lodo de cervejaria, na primeira alimentação. Na segunda alimentação, houve um aumento de 2,4 e 2,7 vezes para o rúmen lavado e o bruto, respectivamente, em comparação com a alimentação anterior. Concluiu-se que o rúmen bovino pode ser utilizado para a 
inoculação de um reator biológico anaeróbio para a degradação de matéria orgânica e produção de metano como biogás.

Palavras-Chave: AME, digestão anaeróbia, inóculo, rúmen.

\section{Introdução}

$\mathrm{O}$ processo de partida, em reatores anaeróbios, sem biomassa de inóculo, tende a ser muito lento e instável (Aquino et al., 2007). Neste sentido, a utilização de inóculo, que é um composto formado por uma série de microrganismos que consomem a matéria orgânica (Lara et al., 2018), constitui-se em uma forma de evitar a falha ou acidificação de um reator anaeróbio.

A Atividade Metanogênica Específica (AME) é uma medida da capacidade e velocidade de conversão de matéria orgânica em metano por um grupo de microrganismos anaeróbios (Aquino et al., 2007). Esta medida é eficiente para a seleção da biomassa de inóculo para reatores anaeróbios, utilizados em estações biológicas de tratamento de efluente (ETE).

O teste de AME é amplamente utilizado na verificação da biodegradabilidade de efluentes neste processo. Liu et al. (2016) utilizaram este teste para comparar a digestão de diferentes substratos, as vias de digestão utilizadas e a influência da concentração dos sólidos na digestão de esgoto doméstico, pela biomassa anaeróbia.

No estudo desenvolvido por TorresLozada \& Pérez-Vidal (2014), o intuito foi averiguar a influência do substrato e a água residuária de extração de amido da mandioca no crescimento e retenção de dois tipos de inóculos diferentes em filtros anaeróbios. Torres et al. (2010) avaliaram a toxicidade de lixiviado puro e misturado com águas residuais domésticas, por meio do teste de AME. Neste estudo, o lixiviado puro provocou redução da $\mathrm{AME}$, tendo sido possível identificar inibições metabólicas e fisiológicas. Rojas et al. (2008) usaram o teste de AME para monitorar o comportamento de uma lama anaeróbia, sob diferentes condições de oxigenação e recirculação de efluente, em um reator aeróbio-anaeróbio de leito fixo no tratamento de esgoto doméstico, observando que o oxigênio não inibiu o comportamento dos organismos metanogênicos. Segundo Alves et al. (2005), a averiguação da inibição de lodo biológico anaeróbio por resíduos de um laboratório de controle de poluição mostrou que os elementos sódio, cromo, fenol e sulfato e as interações sulfato-mercúrio e sulfato-sódio geravam os principais efeitos sobre o percentual de inibição da AME.

O teste de AME avalia a carga orgânica a ser aplicada à biomassa, o desbalanceamento entre as diferentes espécies participantes do sistema anaeróbio, a toxicidade de substâncias aos microrganismos, as rotas da digestão anaeróbia e até o monitoramento de desempenho de sistemas de tratamento.

$\mathrm{O}$ inóculo a ser utilizado em reatores biológicos de tratamento de resíduos pode ser proveniente de várias fontes: reator com biomassa estabilizada, dejeto bovino e suíno, rúmen bovino, entre outros.

Em particular, o rúmen bovino é constantemente objeto de estudo em pesquisas com a digestão anaeróbia, devido a sua alta atividade celulolítica, sendo, geralmente, o inóculo utilizado para o início de processos anaeróbios que não utilizam lodo que tenham operado anteriormente (Quintero et al., 2012).

Os principais fatores que influenciam o crescimento e a atividade dos microrganismos ruminais são: temperatura, $\mathrm{pH}$, capacidade de tamponamento, pressão osmótica e potencial redox (Castillo-González et al., 2014). Sendo assim, durante o teste de AME, é necessário assegurar valores adequados destes parâmetros para que os resultados obtidos não sejam afetados.

As faixas de condições ambientais ótimas para as metanogênicas ruminais variam de 37 a $45^{\circ} \mathrm{C}$ de temperatura, com $\mathrm{pH}$ de 5,9 a 7,4 , visto que cada espécie requer valores específicos dentro destas faixas (Sirohi et al., 2010). Quintero et al. (2012) encontraram, para o rúmen bovino, maior produção de alcalinidade, em comparação a outros inóculos de processos anaeróbios. Desta forma, eles demonstraram uma capacidade de tamponamento mais elevada para o rúmen como inóculo, ainda que, em seu experimento, tenha havido um acúmulo maior de ácidos graxos voláteis do que combinação de rúmen com lama de porco no tratamento de bagaço.

Segundo Castillo-González et al. (2014), os principais produtos finais da fermentação dos nutrientes consumidos pelos microrganismos presentes no rúmen (bactérias, protozoários e fungos) são os ácidos graxos voláteis (AGV) e a biomassa microbiana.

Leite \& Lopes (2001a) utilizaram rúmen como inóculo de reatores de batelada, para bioestabilização de resíduos sólidos e observaram que o rúmen contribuiu para a redução da relação $\mathrm{C}: \mathrm{N}$, evitando que a relação fosse desfavorável. Os autores observaram que uma maior aplicação do inóculo garantia uma melhor eficiência de remoção de Demanda Química de Oxigênio (DQO) $(86 \%$ de remoção, para $15 \%$ em massa de inóculo). Segundo 
Leite \& Lopes (2001b), o inóculo apresentava bactérias anaeróbias que decompunham as cadeias de polímeros até acetato, além de bactérias metanogênicas que convertiam acetato em metano e dióxido de carbono. Foi observado que os valores das constantes de decomposição foram influenciados diretamente pelo percentual de inóculo utilizado, demonstrando que o rúmen bovino tem potencial como inóculo de partida de processos de digestão anaeróbia.

A digestão anaeróbia de palha de milho foi eficiente por microrganismos ruminais, permitindo uma rápida degradação de ácidos voláteis no experimento de $\mathrm{Hu} \& \mathrm{Yu}(2005)$.

A digestão da celulose por microrganismos ruminais é frequentemente avaliada em diferentes estudos; Ruminococcus albus, Ruminococcus flavefaciens e Fibrobacter succinogenes têm altas taxas de presença no rúmen e, em celulose, são capazes de rápido crescimento (Shah et al., 2014).

No tratamento de águas residuais de polpa de café, o rúmen bovino foi capaz de degradar a maior parte da carga orgânica, com $91,2 \%$ de eficiência de remoção de DQO, com pH de 4,6 e $28^{\circ} \mathrm{C}$, em 16 dias (Olvera \& Gutiérrez, 2010).

O valor da AME permite estimar a carga orgânica máxima que pode ser aplicada ao lodo, reduzindo os riscos de desbalanceamento da digestão anaeróbia (Aquino et al., 2007), definindo o tempo de detenção hidráulica e a eficiência esperada. Desta forma, a biomassa de inóculo pode ser selecionada com o auxílio desta técnica, promovendo uma maior eficiência de degradação dos compostos orgânicos e melhor adaptação a choques de cargas orgânicas, o que reforça a importância do conhecimento do valor da AME.
Diante do exposto, o estudo comparou a AME de rúmen bovino com a de uma biomassa de alto desempenho (lodo de estação de tratamento de efluente de cervejaria), seguindo os protocolos propostos por Aquino et al. (2007), nos quais a produção de metano acumulada e a velocidade de produção de diferentes tipos de biomassa podem ser medidas. Por meio deste estudo, pode-se avaliar o potencial de utilização de rúmen bovino como inóculo, em sistemas de tratamento anaeróbio de resíduos biológicos.

\section{Material e Métodos}

Teste de atividade metanogênica específica (AME)

Como reatores, foram utilizadas garrafas de borossilicato (Uniglas), com volume total de $595 \mathrm{~mL}$ e com $10 \%$ de headspace, resultando em um volume útil de $535 \mathrm{~mL}$. Em cada garrafa-reator foram adicionados biomassa, uma solução substrato (fonte de carbono) e uma solução nutriente, conforme apresentado na Figura 1A.

Rúmen lavado (para uma melhor homogeneização) e bruto (Figura 1C) foram testados e comparados com uma biomassa de uma estação de tratamento de efluentes de uma cervejeira (Figura 1B). Segundo Monteggia (1997), a concentração ótima de inóculo é entre 2 e $5 \mathrm{~g} \mathrm{SSV} . \mathrm{L}^{-1}$; a concentração definida para este teste foi de $3 \mathrm{~g} \mathrm{SSV} \cdot \mathrm{L}^{-1}$. A concentração do substrato no teste foi de $6000 \mathrm{mg} \mathrm{O}_{2} \cdot \mathrm{L}^{-1}$ de DQO, e $20 \%$ do volume útil da garrafa reator foi composta de solução nutrientes.

Foram realizadas duas bateladas sequenciais, mantendo a biomassa e trocando apenas o sobrenadante, com as mesmas concentrações descritas acima.
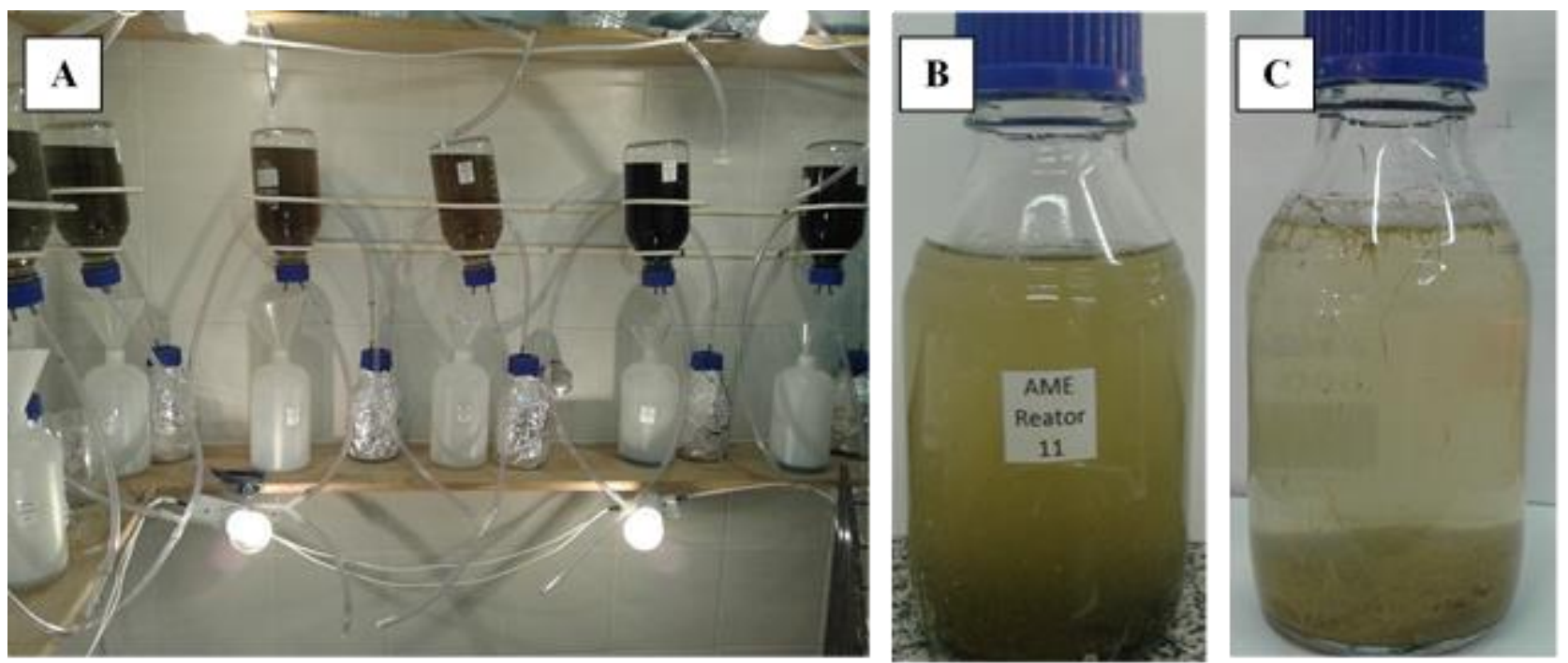

Figura 1. Teste de Atividade Metanogênica Específica (AME). A. aparatos utilizados na execução do experimento; B. garrafa reator com o inóculo de lodo de cervejaria; C. garrafa reator com inóculo de rúmen. Fonte: Pires et al. (2020). 
Após a inoculação do reator e a adição de cada componente, as garrafas permaneceram em uma sala, com temperatura aproximada de $30 \pm$ $3^{\circ} \mathrm{C}$, durante todo o experimento, com agitação manual intermitente a cada 24 horas.

A análise de DQO foi realizada com o sobrenadante dos reatores no início e no final do tempo de operação, com duração de 77 dias para o rúmen lavado, 153 dias para o rúmen bruto e 98 dias para o lodo de cervejaria. O tempo final do experimento foi determinado pela redução na produção do metano (fase estacionária).

\section{Biomassa}

O rúmen bovino foi proveniente de gado de corte alimentado com pasto. O volume de $4 \mathrm{~L}$ foi coletado no abatedouro público da cidade de Afogados da Ingazeira, armazenado em garrafa de plástico e mantido sob refrigeração de $4^{\circ} \mathrm{C}$ até a montagem do teste.

Os microrganismos presentes no rúmen se encontram agregados de forma floculenta com a presença de materiais vegetais. Por isso, o teste de AME foi realizado com o rúmen na forma bruta (sem pré-tratamento) e lavado (Figura 2A). A lavagem do rúmen ocorreu por adição de água potável ao rúmen em uma peneira de 200 mesh de porosidade. $\mathrm{O}$ material retido foi utilizado para inocular os reatores teste. A lavagem é um processo comum para eliminar substrato e padronizar os tamanhos dos flocos. No entanto, como o rúmen não é proveniente de um reator em pleno funcionamento, o teste de AME foi realizado com o rúmen bruto e lavado para verificar se ocorreriam perdas significativas da biomassa ativa.

\section{Lodo de Estação de Tratamento de Efluente (ETE) de cervejaria}

O lodo foi proveniente de um reator Upflow Anaerobic Sludge Blanket (UASB), em operação há um ano e meio, na estação de tratamento de efluentes de uma cervejaria, localizada em Itapissuma (PE). O lodo de cervejaria (Figura 2B) foi lavado (para a remoção de substrato proveniente do reator original e eliminação de pequenos flocos, armazenado em bombonas de plástico e mantido sob refrigeração de $4^{\circ} \mathrm{C}$ até a montagem do teste. Foram realizadas análises de séries de sólidos e de demanda química do oxigênio (DQO) para a caracterização dos inóculos.

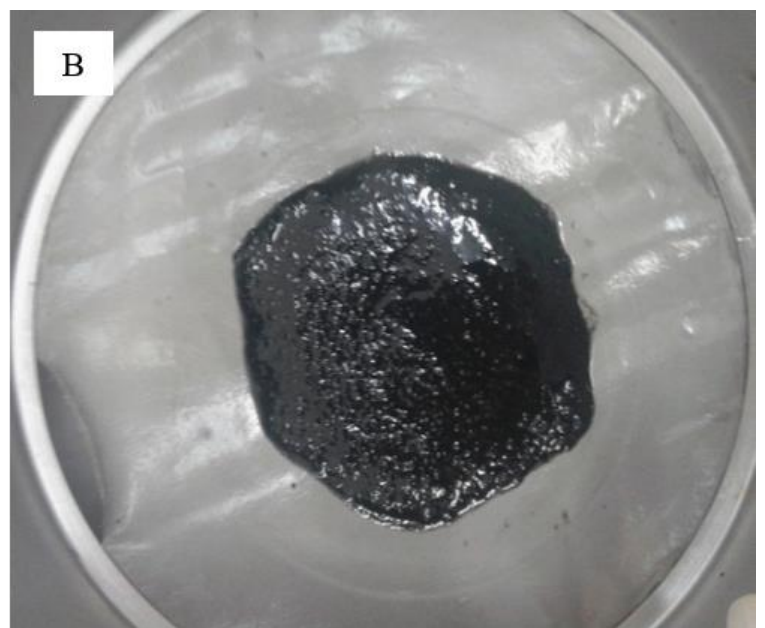

Figura 2. Inóculos. A.) Rúmen bovino lavado para teste de AME; B. Lodo de UASB de ETE de indústria de cervejaria. Fonte: Pires et al. (2020).

\section{Soluções}

A solução de substrato foi uma mistura de três ácidos graxos voláteis: ácido acético (Fmaia, 99\%), ácido propiônico (Sigma-Aldrich, 99\%) e ácido butírico (Vetec, 99\%). A concentração da solução estoque foi de $440 \mathrm{~g} \mathrm{O}_{2} \cdot \mathrm{L}^{-1}$, de DQO, cuja concentração de cada ácido foi de 100:100:100 g.L' ${ }^{1}$ (Aquino et al., 2007).

A solução de nutrientes foi uma mistura de duas soluções, uma de macronutrientes e a outra de micronutrientes, seguindo a composição proposta por Florencio et al. (1993). Este procedimento foi realizado para garantir o tamponamento do sistema massa de bicarbonato de sódio utilizada e respeitou a proporção em massa de 1:1 de bicarbonato:DQO do substrato. Preparou-se $1 \mathrm{~L}$ de solução de hidróxido de sódio $(\mathrm{NaOH})$ a $3 \%$, para cada reator, com indicador de azul de bromotimol para a lavagem do gás produzido.

\section{Análises}

A análise de DQO da alimentação dos reatores foi realizada em triplicata, por meio do método colorimétrico 5220, do Standard Methods for the Examination of Water and Wastewater (Apha, 2012).

A análise de Sólidos Totais (ST) foi realizada em triplicata com os inóculos, por meio 
do método gravimétrico 2540, 2540 B e 2540 E, do Standard Methods for the Examination of Water and Wastewater (Apha, 2012). A análise foi realizada para a medição dos Sólidos Voláteis (SV), visto que os inóculos seriam quantificados pela concentração por litro, indicada na literatura.

$\mathrm{O}$ biogás produzido nos reatores foi conduzido por mangueiras de PVC para uma garrafa contendo uma solução de hidróxido de sódio $(\mathrm{NaOH})$ a $3 \%$. Esta solução captura o gás carbônico e a água presente no biogás, deixando apenas o metano no headspace. Um volume da solução de hidróxido era deslocado para fora da garrafa, referente a um volume semelhante de metano que entrava nela. Este deslocamento foi quantificado diariamente e convertido em volume produzido de metano (Aquino et al., 2007).

\section{Cálculo da AME}

Ao plotar o volume de metano acumulado pelo tempo, calculou-se a inclinação da reta da fase $\log$, fase de produção linear de metano. $\mathrm{O}$ valor desta taxa é utilizado para calcular a AME, seguindo a Equação 1 (Aquino et al., 2007).

$$
A M E=\frac{\Delta C H_{4}}{\Delta t} * \frac{1}{V * C_{i} * 394}
$$

onde: $\frac{\Delta \boldsymbol{C H}_{4}}{\Delta \boldsymbol{t}}$ representa a taxa máxima de produção de metano obtida pela inclinação da reta no gráfico da produção acumulada (mLDQO- $\left.\mathrm{CH}_{4} \cdot \mathrm{d}^{-1}\right), V$ é o volume útil do reator (em $\mathrm{L}), C_{i}$ é a concentração do inóculo (g SSV.L ${ }^{-1}$ ) e o valor 394 representa a correção do cálculo de AME (1 g DQO produz um valor de $394 \mathrm{~mL}$ de metano, a $30^{\circ} \mathrm{C}$ ).

\section{Resultados e Discussão}

Os resultados da série de sólidos são apresentados na Tabela 1. A análise gravimétrica da série de sólidos para os inóculos rúmen bruto e lavado mostrou que o procedimento de lavagem do inóculo removeu partículas inorgânicas e não afetou a concentração média dos compostos orgânicos. Observou-se que o rúmen bovino apresentou concentração de sólidos voláteis maior que o lodo de cervejaria.

Tabela 1. Concentração média e desvios padrão dos sólidos totais, inorgânicos (Fixo) e orgânico (volátil) nos inóculos (lodo e rúmen) in natura (bruto) e após a elutriação (processo de lavagem com as peneiras). Pires et al. (2020).

\begin{tabular}{lccc}
\hline Inóculo & Sólidos Totais & Sólidos Totais Fixos & Sólidos Totais Voláteis \\
\hline Rúmen bruto & & $\left(\mathbf{m g . L}^{-1}\right)$ & \\
Rúmen lavado & $143 \pm 9,00$ & $14,2 \pm 0,50$ & $129 \pm 9,00$ \\
Lodo de cervejaria bruto & $143 \pm 2,00$ & $7,75 \pm 0,09$ & $135 \pm 2,00$ \\
Lodo de cervejaria lavado & $94 \pm 3,00$ & $18,1 \pm 0,40$ & $76 \pm 2,00$ \\
\hline
\end{tabular}

*procedimento padrão de pré-tratamento da biomassa para a remoção de substrato e regularização do tamanho dos flocos ou grânulos.

O rúmen apresenta, em sua composição, pedaços de vegetais parcialmente digeridos. Estes pedaços são orgânicos, o que pode contribuir com a concentração mais elevada de sólidos orgânicos no rúmen, em comparação ao lodo de cervejaria. Outro fator a ser considerado é que estes pedaços vegetais podem funcionar como meio de suporte para o crescimento e a fixação de colônias de microrganismos, reduzindo seu carreamento na lavagem. Podem-se considerar os valores médios da concentração dos sólidos voláteis semelhantes, estatisticamente, entre os rúmenes bruto e o lavado. Isto ocorreu porque a concentração das réplicas para o rúmen bruto variou de $9 \mathrm{mg}$ para mais e para menos em relação ao valor médio, enquanto, para o rúmen lavado, a variação foi de $2 \mathrm{mg}$. A variação foi maior no rúmen bruto devido a distribuição mais heterogênica das colônias de microrganismos suspensas.

O processo de lavagem da biomassa é uma rotina de laboratório para eliminar os microrganismos desprendidos dos flocos (suspensos) e de compostos provenientes dos afluentes iniciais dos sistemas de onde foram coletados. Estes compostos orgânicos podem alterar o valor da DQO adicionada, comprometendo a comparação entre os cálculos teóricos e práticos. No processo de inoculação, a fração volátil (orgânica) dos sólidos é relacionada diretamente com a quantidade de microrganismos.

Na Tabela 2 são apresentados os resultados obtidos para o teste de AME, a quantidade de matéria orgânica degradada, em termos de DQO, e o tempo de operação de cada reator, em cada alimentação. 
Tabela 2. Resultados do teste de Atividade Metanogênica Específica (AME): taxa de degradação da matéria orgânica pelos microrganismos (AME), quantitativo de matéria orgânica degradada e tempo de incubação de cada batelada sequencial. Fonte: Pires et al. (2020).

\begin{tabular}{|c|c|c|c|c|c|c|}
\hline \multirow{2}{*}{$\begin{array}{l}\text { Alimentação } \\
\text { Inóculo }\end{array}$} & \multicolumn{2}{|c|}{$\begin{array}{c}\text { AME } \\
\left(\mathrm{g} \mathrm{DQO}_{\mathrm{CH} 4 . \mathrm{g} \mathrm{SSV}}^{-1} \cdot \mathrm{d}^{-1}\right)\end{array}$} & \multicolumn{2}{|c|}{$\begin{array}{c}\text { DQO degradada } \\
\left(\mathrm{g} \mathrm{O}_{2} \cdot \mathrm{L}^{-1}\right)\end{array}$} & \multicolumn{2}{|c|}{$\begin{array}{c}\text { Tempo de operação do } \\
\text { reatores (dias) }\end{array}$} \\
\hline & $1^{\mathrm{a}}$ & $\mathbf{2}^{\mathrm{a}}$ & $1^{\mathrm{a}}$ & $\mathbf{2}^{\mathrm{a}}$ & $\mathbf{1}^{\mathbf{a}}$ & $2^{\mathbf{a}}$ \\
\hline Rúmen lavado & $0,0115 \pm 0,0007$ & $\begin{array}{c}0,028 \pm \\
0,006\end{array}$ & $3,99 \pm 0,01$ & $4,9 \pm 0,4$ & 50 & 27 \\
\hline Rúmen bruto & $0,025 \pm 0,001$ & $\begin{array}{c}0,069 \pm \\
0,008\end{array}$ & $4,9 \pm 0,40$ & $4,3 \pm 0,2$ & 105 & 48 \\
\hline Lodo de cervejaria & $0,104 \pm 0,004$ & $\begin{array}{c}0,025 \pm \\
0,002\end{array}$ & $3,14 \pm 0,09$ & $4,9 \pm 0,2$ & 38 & 60 \\
\hline
\end{tabular}

Observou-se que foi necessário um tempo diferente de operação dos reatores para cada tipo de inóculo, devido ao desempenho particular de cada um.

O lodo de cervejaria, apesar de menor concentração de sólidos voláteis, mostrou melhor desempenho na produção de metano que os rúmenes testados. A AME do lodo de cervejaria foi de quatro e nove vezes mais elevada que a do rúmen bruto e do lavado, respectivamente. O lodo de cervejaria se mostrou adaptado a elevadas cargas orgânicas, com altas concentrações de DQO. Segundo a literatura, a concentração de DQO de efluentes de cervejaria pode variar entre 1000 e 12000 mg.L. ${ }^{-1}$ (Inyang et al., 2012; Emitan et al., 2014; Rocha et al., 2017). A composição deste tipo de efluente é normalmente formada por compostos mais complexos, com grandes cadeias carbônicas. O rúmen, por sua vez, tem um grupo de microrganismos adaptados a digestão de vegetais, que é uma biomassa lignocelulósica e altamente recalcitrante à degradação anaeróbica, por ser composta de celulose, hemicelulose e ligninas (Surendra \& Khanal, 2015).

Neste experimento, o lodo de cervejaria e o rúmen receberam uma mistura de ácidos graxos voláteis, um substrato utilizado em testes para avaliar a atividade dos microrganismos metanogênicos e a atividade dos microrganismos sintróficos, responsáveis por converterem propionato e butirato em acetato (Aquino et al., 2007). Assim, a baixa produção de metano dos rúmenes testados pode estar relacionada à atividade dos microrganismos sintróficos produtores de acetato e não à atividade dos microrganismos metanogênicos que consomem o acetato para transformá-lo em metano.

Na segunda alimentação, observou-se uma redução na atividade do lodo de cervejaria de quatro vezes e um aumento de 2,5 e 2,7 vezes para o rúmen lavado e o bruto, respectivamente. Os dois tipos de inóculos foram submetidos a condições hidrodinâmicas muito distintas das condições originais, onde as colônias foram formadas. Esta mudança nas condições hidrodinâmicas pode ser um dos fatores que favoreceu o desenvolvimento e a atividade do rúmen $\mathrm{e}$ ter prejudicado o desempenho do lodo de cervejaria. O rúmen bruto, seguido pelo lavado, degradou mais matéria orgânica que o lodo de cervejaria, na primeira alimentação. Todavia, a produção do metano deuse de forma mais lenta.

Xiangwen et al. (2008) avaliaram a AME da biomassa de cervejaria em um reator piloto e, na primeira alimentação, os valores médios foram de $0,11 \pm 0,04$, resultado semelhante ao encontrado na primeira alimentação deste estudo. Após 60 dias de operação, e com o lodo completamente granular, a AME aumentou para 0,53 $\pm 0,19$ g DOQ.g SSV ${ }^{-}$ ${ }^{1} . \mathrm{d}^{-1}$, estando estes reatores anaeróbios em regime de batelada sequencial e alimentados com uma concentração de matéria orgânica de $5000 \mathrm{mg}$ DQO.L ${ }^{-1}$. Este comportamento foi diferente do encontrado neste estudo, onde o lodo de cervejaria reduziu sua velocidade de degradação, apesar da concentração de matéria orgânica e da dinâmica de reator terem sido semelhantes.

Os valores de AME do lodo de cervejaria, encontrados na primeira alimentação, podem ser considerados satisfatórios e coerentes para a atividade descrita em alguns estudos com lodos industriais. Silva et al. (2013) realizaram o teste com dois inóculos: lodo têxtil (aeróbio), coletado na entrada de um decantador biológico secundário de sistema de lodos ativados, e encontraram um valor de AME de 0,10 g DQO-CH4 g SSV.d ${ }^{-1}$, com uma produção de $3,04 \mathrm{~mL}$ de metano, e lodo alimentício, coletado de um reator UASB anaeróbio, com 0,17 g DQO- $\mathrm{CH}_{4}$ g SSV.d ${ }^{-1}$, e produção de $337,05 \mathrm{~mL}$ de metano.

No tratamento de águas residuais de polpa de café, utilizando rúmen bovino como inóculo, foi encontrado um valor de AME de 0,0076 g DQO G SST $^{-1} \mathrm{~d}^{-1}$ (Olvera \& Gutiérrez, 2010), valor inferior ao encontrado nas duas alimentações deste estudo. Pérez et al. (2000) utilizaram dois inóculos diferentes: esterco bovino e lama de lagoas de estabilização, no tratamento de águas residuais de 
polpa de café, obtendo melhores resultados de

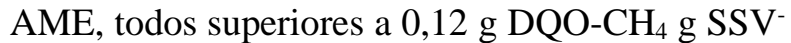
${ }^{1} \mathrm{~d}^{-1}$ e superiores aos valores encontrados neste estudo.

A Figura 3 mostra a produção de metano nos reatores alimentados com o rúmen lavado, com o rúmen bruto e com o lodo de cervejaria, na $1^{\mathrm{a}} \mathrm{e}$ $2^{\mathrm{a}}$ alimentação. Observa-se que o rúmen precisou de mais tempo de adaptação (fase lag) que o lodo de cervejaria, na primeira alimentação. $\mathrm{Na} 2^{\mathrm{a}}$ alimentação, esta fase foi reduzida para os três inóculos, demonstrando um aumento da capacidade de degradação do rúmen. Nota-se que a lavagem do rúmen bovino pode ter afetado o valor da AME (Figura $3 \mathrm{~A}$ ).

Em 50 dias de operação dos reatores, com inóculo lavado na primeira alimentação, foi produzida uma média de $120 \mathrm{~mL}$ de metano; na realimentação foram produzidos $164 \mathrm{~mL}$ em 27 dias de operação. Na primeira alimentação do teste com o rúmen bruto obteve-se uma média de 536 $\mathrm{mL}$ de metano produzidos em 105 dias, na realimentação, em apenas 48 dias de operação obteve-se uma média de $812 \mathrm{~mL}$.
Esses resultados ainda são inferiores ao do lodo de ETE de cervejaria, que produziu $890 \mathrm{~mL}$ e $620 \mathrm{~mL}$ de metano em 38 e 60 dias, na primeira e segunda alimentação, respectivamente. A análise de sólidos mostrou uma pequena diferença entre a concentração de sólidos voláteis do rúmen bruto e lavado.

Apesar de o reator com o rúmen lavado ter sido operado em menor tempo, pode-se observar que, no mesmo tempo de operação, as produções e velocidades foram distintas.

O lodo de cervejaria necessitou de pouco tempo de adaptação para atingir a velocidade máxima; o rúmen bruto foi o que precisou de maior tempo de adaptação e os resultados obtidos, após uma segunda alimentação, foram melhores em termos de velocidade. Logo, um período de 50 dias foi suficiente para promover adaptação da biomassa em todos os reatores.

No entanto, o processo de lavagem removeu a biomassa livre, isto é, não aderida nos pedaços de palha ou agregados em flocos. A remoção da biomassa livre, provavelmente, foi a causa da redução nos valores de AME com o rúmen lavado, promovendo a perda de biomassa ativa.

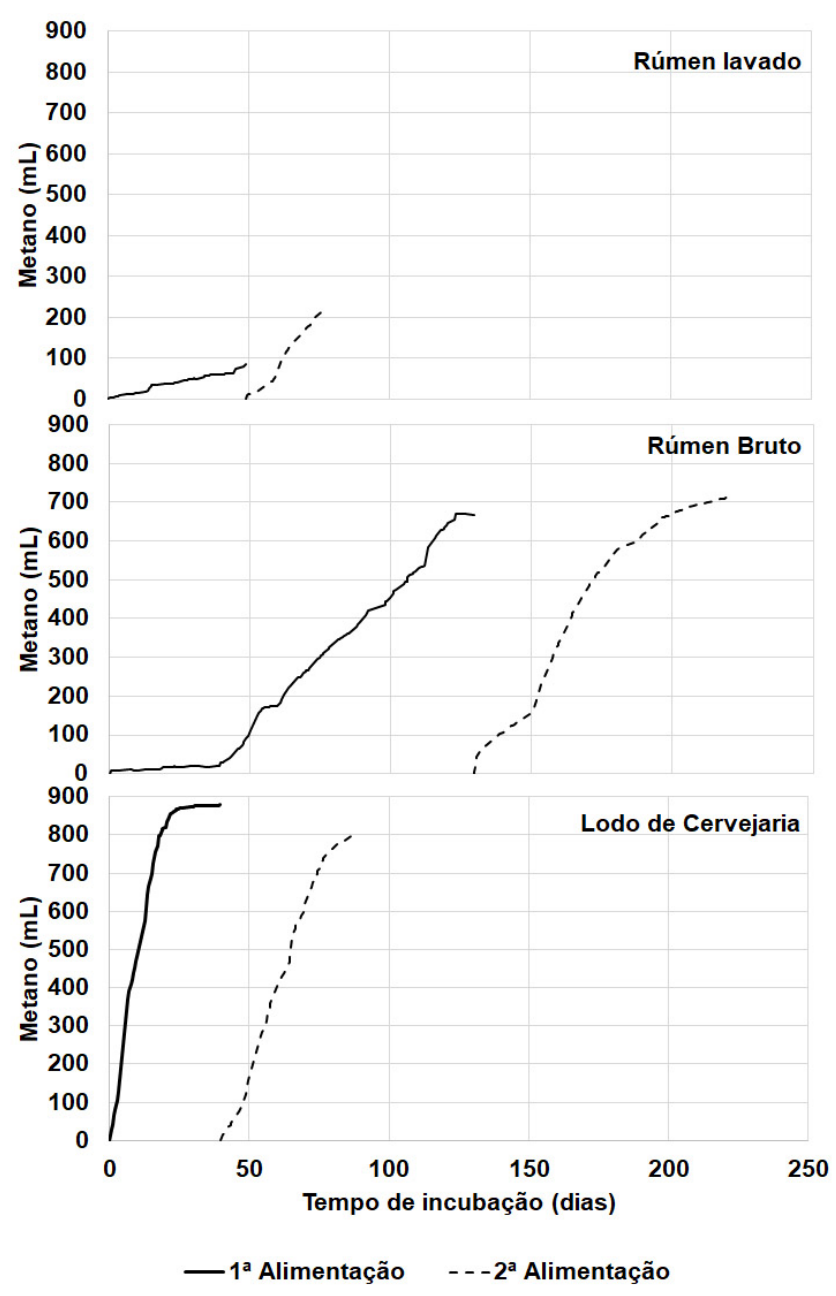

Figura 3. Produção de metano ao longo do tempo de incubação nos reatores inoculados com rúmen lavado, rúmen bruto e lodo de cervejaria, na primeira e segunda alimentação. Fonte: Pires et al. (2020). 
A DQO consumida não correspondeu à quantidade de metano produzido. A literatura registra que $1 \mathrm{~g}$ de DQO produziria $394 \mathrm{~mL}$ de metano (Aquino et al., 2007). Valores inferiores a este foram obtidos com todos os inóculos. A relação metano produzido.DQO ${ }^{-1}$ consumida era maior, quanto melhor fosse o desempenho do inóculo, isto é, quanto maior fosse o valor de AME. É provável que outras rotas de consumo de DQO tenham ocorrido, como aquelas de redutoras de sulfato, talvez pela contaminação dos inóculos.

\section{Conclusão}

O rúmen bovino mostrou elevado potencial como inóculo para reatores anaeróbios de tratamento de resíduos. O rúmen apresentou resultados satisfatórios de degradação de matéria orgânica, e adaptação as condições deste com o aumento da atividade, em relação a alimentações anteriores.

Os microrganismos mais ativos foram encontrados na biomassa suspensa e, por isso, o rúmen, após processo de elutriação (lavagem), perdeu biomassa ativa, contribuindo com a redução da velocidade de degradação da matéria orgânica disponível.

Desta forma, pode-se concluir que o rúmen pode ser utilizado para a inoculação de um reator biológico anaeróbio para a degradação de matéria orgânica e a produção de metano como biogás.

\section{Agradecimentos}

Os autores agradecem à Pró-Reitoria para Assuntos de Pesquisa e Pós-Graduação (Propesq/UFPE) pela bolsa de iniciação científica concedida à Rachel Barros Pires e à Coordenação de Aperfeiçoamento de Pessoal de Nível Superior (CAPES), pelo apoio financeiro ao Programa de Pós-Graduação em Engenharia Civil e Ambiental.

\section{Referências}

Apha. 2012. Standard Methods for the Examination of Water and Wastewater, 22th ed. Washington. 2671p.

Alves, L. C.; Cammarota, M. C.; França, F. P. 2005. Inibição de lodo biológico anaeróbio por constituintes de efluente de laboratório de controle de poluição. Engenharia Sanitária e Ambiental, 10, (3), 236-242. doi: 10.1590/s1413-41522005000300008.

Aquino, S. F.; Chernicharo, C. A. L.; Foresti, E.; Florêncio, M. L. S.; Monteggia, L. O. 2007. Metodologias para determinação da atividade metanogênica específica (AME) em lodos anaeróbios. Engenharia Sanitária e Ambiental, 12, (2), 192-201. doi: 10.1590/s1413-41522007000200010.
Castillo-González, A. R.; Domínguez, V. J.; Burrola-Barraza, M. E.; Chávez-Martinez, A. 2014. Rumen microorganisms and fermentation. Archivos de Medicina Veterinaria, 46, (3), 349-361. doi: 10.4067/s0301-732x2014000300003.

Emitan, A. M.; Swalaha, F. M.; Adeyemo, J.; Bux, F. 2014. Assessment of brewery efluente composition from a beer producing industry in Kwazulu-Natal, South Africa. Fresenius Environmental Bulletin, 23, (3), 693-701.

Florencio, L.; Jenicek, P.; Field, J. A.; Lettinga, G. 1993. Effect of Cobalto $n$ the anaerobic degradation of metanol. Journal of Fermentation and Bioengineering, 75, (5), 368-374.

Hu, Z. H.; Yu, H. Q. 2005. Application of rumen microorganisms for enhanced anaerobic fermentation of corn stover. Process Biochemistry, 40, (7), 2371-2377. doi: 10.1016/j.procbio.2004.09.021.

Inyang, U. E.; Bassey, E. N.; Inyang, J. D. 2012. Characterization of Brewery effluent fluid. Journal of Engineering and Applied Sciences, 4, 67-77.

Leite, V. D.; Lopes, W. S.; Prasad, S. 2001a. Bioestabilização anaeróbia de resíduos sólidos orgânicos em reatores de batelada. Revista Brasileira de Engenharia Agrícola e Ambiental, 5, (1), 119-123.

Leite, V. D.; Lopes, W. S.; Prasad, S. 2001b. Influência do Inóculo no Processo de Tratamento Anaeróbio de Resíduos Sólidos Orgânicos. $21^{\circ}$ Congresso Brasileiro de Engenharia Sanitária e Ambiental. João Pessoa-PB. 39p.

Liu, C.; Li, H.; Zhang, Y.; Chen, Q. 2016. Characterization of methanogenic activity during high-solids anaerobic digestion of sewage sludge. Biochemical Engineering Journal, 109, 96-100.

Lara, M. S. M.; Lizaolab, R. Q.; Espinosa Victoria, D.; Alarcónb, A.; Barrab, J. D. E.; Santosb, A. T.; Martínez, F. V. C. 2018. Generación de un inoculante acelerador del compostaje. Revista Argentina de Microbiolía, 50, (2), 206-210.

Monteggia, L. 1997. Proposta de metodologia para avaliação do parâmetro Atividade Metanogênica Específica. $19^{\circ}$ Congresso Brasileiro de Engenharia Sanitária e Ambiental, ABES, Foz do Iguaçu. pp. 754766.

Olvera, J. R.; Gutiérrez, J. I. 2010. Biodegradación anaerobia de las aguas generadas en el despulpado del café. Revista Colombiana de Biotecnología, 12, (2), 230-239. 
Pérez, R. M.; Rodríguez, S.; Fernández, M. 2016. Estudio de la biodegradabilidad anaerobia de las aguas residuales del beneficio húmedo del café Interciencia [en linea], 2000, 25. Disponível em: <http://www.redalyc.org/articulo.oa?id=339 05005>.

Quintero, M.; Castro, L.; Ortiz, C.; Guzmán, C.; Escalante, H. 2012. Enhancement of starting up anaerobic digestion of lignocellulosic substrate: fique's bagasse as an example. Bioresource Technology, 108, 8-13.

Rocha, C. M; Machado, K. S. C.; Andrade, L. H. Brandt, E. M. F. 2017. Dimensionamento e comparação de estação de tratamento de efluentes utilizando lodos ativados e reator UASB para uma cervejaria artesanal. Revista Processos Químicos, 11, (22), 83-85.

Rojas, M. P. A.; Netto, A. P. O.; Zaiat, M. 2008. Actividad metanogénica específica en un reactor anaerobio - aerobio aplicado al tratamiento de agua residual doméstica. Interciencia, 33, 4, 284-289.

Shah, F. A.; Mahmood, Q.; Shah, M. M.; Pervez, A.; Asad, A. 2014. Microbial Ecology of Anaerobic Digesters: The Key Players of Anaerobiosis. The Scientific World Journal, 2014, 1-21. doi: 10.1155/2014/183752.

Silva, J. D.; Schneiders, D.; Till, A.; Lapa, K. R.; Pinheiro, A. 2013. Atividade metanogênica específica (AME) de lodos industriais provenientes do tratamento biológico aeróbio e anaeróbio. Ambi-Agua, 8, 2, 135-145. doi: 10.4136/ambi-agua.1098.

Sirohi, S. K.; Pandey, N.; Singh, B.; Puniya, A. K. 2010. Rumen methanogens: a review. Indian J. Microbiol., 50, (3), 253-262. Doi: 10.1007/s12088-010-0061-6.

Surendra, K. C.; Khanal, S. K. 2015. Effects of crop maturity and size reduction on digestibility and methane yield of dedicated energy crop. Bioresource Technology, 178, 187-193. doi: 10.1016/j.biortech.2014.09.055

Torres-Lozada, P.; Pérez-Vidal, A. 2014. Influence of inoculum on growth and retention of the biomass in anaerobic filters. Revista Facultad de Ingeniería Universidad de Antioquia, 72, 127-133.

Torres, P.; Barba, L. E.; Pizarro, C. 2010. Mitigación de la toxicidad anaerobia de lixiviados mediante mezclas con agua residual doméstica. Rev. Fac. Ing. Univ. Antioquia, 53, 64-74.

Xiangwen, S.; Dangcong, P.; Zhaohua, T.; Xinghua, J. 2008. Treatment of brewery wastewater using anaerobic sequencing batch reactor (ASBR). Bioresource Technology, 99, 3182-3186. 